\title{
A global perspective on capsule endoscopy
}

\author{
Tanja Nowak ${ }^{1,2}$ \\ ${ }^{1}$ Master Program MHMM (Health and Medical Management), Friedrich-Alexander-University Erlangen-Nuremberg, Germany; ${ }^{2}$ Consultant \\ Medical Affairs, Hamburg, Germany \\ Correspondence to: Dr. Tanja Nowak, PhD. Max-Brauer-Allee 100, D-22765 Hamburg, Germany. Email: tanja.nowak@medical-affairs.de.
}

\begin{abstract}
A search of the internet today to quantify the estimated value of capsules from a global perspective, easily delivers figures stating around \$200 million in 2014 to about \$400 million by 2020, which would be approximately $10 \%$ of the gastrointestinal endoscopic market. Is this a steep rise within just six years or could the capsule market do even better? What chances does this offer and what are the key aspects for future success? By 2020, more than 1 billion people are aged sixty or older and around one third of them will live in what the UN calls "more developed regions". Naturally, this brings an increased demand for colorectal cancer screening and surgery. But keeping in mind that basically every healthcare system, in any country, is already operating at its limits, how do we secure future treatment for a growing community? Surely more competition will steadily bring down prices for capsules. However, that does not ease the amount of time that is spent to properly read any video and issue a valid diagnosis for every patient. This article intends to give an overview about the current global market for capsule endoscopy (CE) with a perspective on typical patients, their indications, and how the capsules are used and by whom. Further aspects, such as standardization of training, reading and future trends will also be elaborated on.
\end{abstract}

Keywords: Capsule endoscopy (CE); remote capsule service; home delivery; pan-intestinal capsule; e-health; Crohn's disease; inflammatory bowel disease (IBD); centralized reading

Submitted Feb 16, 2017. Accepted for publication Jul 24, 2017.

doi: $10.21037 /$ atm.2017.10.20

View this article at: http://dx.doi.org/10.21037/atm.2017.10.20

\section{Global market-overview}

Ahead of discussing possible future market trends, a closer investigation of the current state of capsule endoscopy (CE) is mandatory. The market can be described from different perspectives: first, the technological progress which includes different capsule types and technical developments. Second, different indications are established or planned. The two last perspectives concern general market access and how that is enabled or constraint by reimbursement policies. The latter is of most interest to those who pay the bill: the patients themselves, society and those who develop, enhance and distribute new technologies-the manufacturers and service providers.

\section{Capsule types and technology}

First video capsule ever was called "M2A" (mouth to anus), and was launched in 2001 by Given Imaging Ltd. M2A was intended to be used as a "whole gut" capsule. However it turned out that this first capsule was best used to visualize the small bowel (SB), which was a "black box" at that time. The company renamed the product PillCam $\mathrm{SB}^{\circledast}$ (small bowel) capsule and provided units to 2 million patients by 2014. Meanwhile, Given Imaging resides within Medtronic and the portfolio has expanded to include other capsule types [UGI (upper GI), colon, SBC (small bowel colon)]. Other manufacturers, such as Ankon Technologies, CapsoVision, Chongqing Jinshan Science \& Technology, IntroMedic and Olympus, with further technical developments such as magnetic guidance entered the market (1-3).

Not only have the optical lenses and image resolutions improved, but also the software has constantly advanced. The resolution of modern capsule images is at a maximum $512 \times 512$ pixel (4). With the help of software algorithms 
picture quality is enhanced to provide enough details for all relevant findings. Additional tools like a suspected blood indicator (5), Lewis Score for evaluating Crohn's disease (6) and reading "accelerators" are available (7). Localization or $3 \mathrm{D}$ imaging is one key parameter to guide follow-up procedure, new techniques are currently under development (8).

No commercially available capsule is capable of taking biopsies, but a polyp, tumor or other relevant findings should be detected with high precision (9). Practice suggests that most limitations in the use of CE come from inadequate bowel preparation especially when visualizing the large bowel. Due to the regular application of a cleansing scale (10) in video reports, however, it is obvious that currently available regimens can yield good results (9).

Other technical approaches are described, e.g., an X-ray imaging capsule aiming for a prep-less visualization of the colon (11). A Spanish team developed the so-called Endoluminal Image Analysis (EIA) which is supposed to capture intestinal motility information due to changing patterns during the peristaltic waves in ill versus healthy patients (12). A completely different technology is available with the wireless motility capsule which aims to provide information on transit times, $\mathrm{pH}$ and pressure parameters but no visualization of the mucosa (13). Furthermore, concepts and projects such as EndoVESPA and Sonopill are subject to further articles in this edition.

\section{Indications of CE}

The most used capsule so far is targeting the SB where it is seen as gold standard if GI bleeding is suspected, but nothing was found during upper and lower endoscopy (14). Rockey describes that "occult gastrointestinal bleeding [defined as bleeding that is unknown to the patient] manifests as fecal occult blood and/or iron deficiency anemia (IDA); it is extremely common and can be caused by virtually any lesion in the gastrointestinal tract" (15). Depending on the bleeding sources, a video capsule might detect miscellaneous findings varying from angioectasia to tumors, from ulcers to abnormal mucosal villi. In some patients, a screening for intestinal blood loss may even help to identify SB metastasis of malignant melanoma (16).

Different capsules enlarge the variety of utilization and diagnostics. A team from London described findings like hiatus hernia, esophagitis and erosive gastritis in the upper part (17), whereas polyps, cancer, Crohn's disease lesions, diverticulitis, and others can be found in the lower part of the gut (18).

Colon capsule endoscopy (CCE) allows the visualization of the colon from proximal to distal while colonoscopy, the current gold standard for colon examination, moves anticlockwise. This procedural difference is utilized in the case of unsuccessful colonoscopies, procedures where the endoscope did not reach the cecum. A capsule is regularly able to visualize the unseen segment as shown in clinical trials $(19,20)$ and in daily clinical routine (21). In 2012, this was one indication that was recommended by the ESGE that published a comprehensive guideline (22). Consequently, adequate additional trials were initiated, and meanwhile, the FDA approved CCE for patients with "incomplete colonoscopy" and "at major risks for colonoscopy or moderate sedation" in the US (23). This is similar to the current situation in Japan (PMDA) (24). Aside from incomplete colonoscopy, the ESGE mentions that "CCE is feasible and safe and appears to be accurate when used in averagerisk individuals". By this, CCE could be an additional tool in colorectal cancer screening. However, its widespread implementation is not only a question of study data or consecutive results, but much more subject to acceptance, clinical pathways and a proof of cost-effectiveness.

Study data show that capsules can also be used for a pan-intestinal videography, which makes it attractive as a tool in patients with inflammatory bowel disease (IBD). This is valid for Crohn's disease (25) as well as Ulcerative Colitis (26), depending on the patient profile, age and the overall needs, e.g., to identify the location and extension of the disease (27) and to monitor the activity of the disease (28) and treatment effectiveness or to rule-out Crohn's disease (29).

While the focus may vary, capsules are used for patients from 2 years and upwards, as documented in regulatory approvals such as CE-Mark, with a few contraindications to be respected (30).

\section{Reimbursement and access to CE}

The first capsules have been used in trials only. Based on the safe and minimally invasive character plus the excellent diagnostic yield, at least the SB capsule in its indication "obscure GI bleeding" is licensed and reimbursed in many countries all over the world such as Portugal as described by Bruno Rosa (31). Nevertheless, despite variety of indications, a standardized procedural and reading approach is important. Based on the principle of the MST (Minimal Standard Terminology) for endoscopy, the terminology of SB CE has been categorized, called CEST (Capsule 
Endoscopy Standard Terminology) (32).

Training is strongly advised or even mandatory according to local regulations. Within the USA the ASGE (33) provides consistence guidance, while across Europe medical education of GIs (34) differs. In Germany, e.g., the public health system states that a capsule training is mandatory for office-based GIs to receive reimbursement for a SB CE. Wherever applicable, experienced capsule users provide theoretical and practical training on beginners and advanced levels $(35,36)$, supported by manufacturers or societies $(37,38)$. Moreover an international team from Europe elaborated on parameters to improve and standardize training, by providing a core curriculum (39).

A comprehensive e-learning tool is available for CCE as described by Watabe et al. (40), and should be available for other capsule types in the near future as stated by Kattrina Richardson, Group Manager, Global Market Development at Medtronic. Dedicated associations like the Japanese Association of Capsule Endoscopy (JACE) are aiming to deliver standards for capsule training and reading for the use in both the SB and colon (41).

In few regions, the regulatory process is highly complex and time consuming and need support by specialized local companies. Therefore, capsules are offered only to patients, if they can afford it privately, e.g., in Russia.

The manufacturers' perspective is always driven by turnover. They tend to observe the market for innovations which hit the needs of many. This happened with the invention of the SB capsule, where a novel product closed the gap on directly visualizing the mucosa in the small intestine. As of today, when considering the large intestine, optical colonoscopy is an established procedure and seen as the undisputed reference standard. Thereby, CCE still faces a niche existence for patients where colonoscopy reaches its limits.

While some countries start to walk down a different path, new business opportunities are emerging. Long waiting lists in Australia (42), Denmark, UK etc. and limited capacities, as well as the knowledge that screening programs help save lives in the same way that they avoid costs associated with belated treatment, has opened the door for pilot projects. In Germany, the awareness for colon cancer is regularly brought back to mind and one of the biggest public health insurances (AOK Bavaria) started to offer colon capsules for CRC screening as an (additional) option to colonoscopy in a pre-defined geographical area (43). Here, an interventional colonoscopy is even offered on the same day, when a capsule video revealed findings (44). Overall results of the project are expected soon.

Denmark followed with the "CareforColon" project, where in multiple clinical trials patients are offered CCE that is delivered as a fully outsourced, end-to-end "CCE-service" by CorporateHealth International. This service comprises of home service nurses carefully instructing patients and supervising the CCE procedure at home or an office. This is being followed by the electronic transfer of the (anonymized) video to a central reading site where experts evaluate the results and return a report to the responsible doctor within 3 days. A key element for the seamless and cost effective service delivery is the use of a newly designed IT platform. For the Danish project, the national health portal Sundhed.dk provides the fully compliant linkage into the electronic health record for each patient (45). Thus, even a home-delivered CCE procedure and centralized video evaluation has become feasible. During the first phase trial (46), a colon capsule was offered after positive FIT (fecal immunochemical test) before sending patients to colonoscopy. The aim was to evaluate the efficiency of CCE in the detection of neoplasms compared to conventional colonoscopy to understand its role as a filter test. Manuscript is submitted.

Another reason why CCE might find more market traction is because of its role in risk management. A recent paper elucidates that eighty-five percent of endoscopes required repair due to findings like blood, tissue and bacteria (47). This is an alarming aspect translating into considerable increased risk of contamination and costs which are difficult to display in a reimbursement dossier. Living in the $21^{\text {st }}$ century and having in mind, that multiresistant germs like MRSA are observed to spread, it is eligible to question if simple screening of low-risk or healthy individuals should be still subject to precious medical capacities or if comprehensive, minimally invasive single use tools may be allowed to ease the pressure on extensive waiting lists, while they help as well in minimizing the risk of infections.

\section{Future trends}

A major benefit of CCE (see Table 1) is that videos can be stored, transferred and reviewed, as part of the patients' record. Therefore, a review of any segment is possible at any time, while in standard practice for colonoscopy or ultrasound only a few snapshots are saved. While technological breakthrough of automated video reading is still being sought, manual analysis is required. The goal for 
Table 1 Overview on capsule features

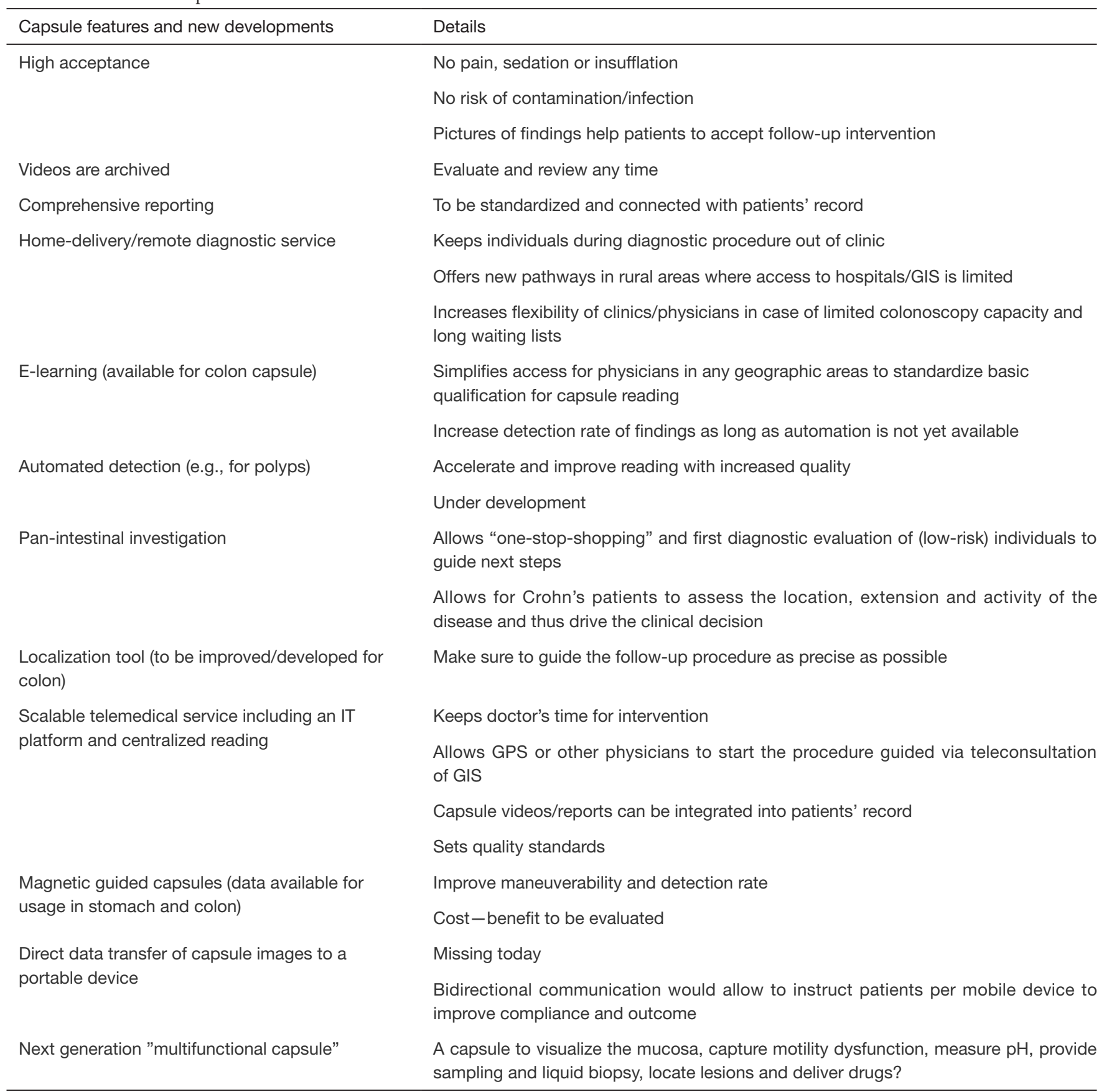


an easy use of capsules in clinical routine is a quick reading of the whole video without missing relevant findings. Since there is no standardized approach, in some regions, nurses play an important role and support doctors not only with uploading the data but also with pre-reading (48). Here, a systematic, scalable approach of centralized reading embedded into a sophisticated IT platform becomes part of the solution.

Centralized reading services might increase the quality as well as improve time management and flexibility, especially in hospitals where capsule reading is rare and where there are no dedicated specialists. As well-from the holistic point of view-this might also open capsule diagnostics to general practitioners or other medical doctors, who could initiate a capsule procedure with the aim to refer only patients with findings to the GI. This would accomplish the idea of a remote diagnostic tool which was already discussed in 2015 (49).

Diagnostic tools should be chosen according to the patient's profile and (suspected) indication. With the new generation of mobile devices and physicians who are familiar with e-learning, e-health and electronic patient records, the classical treatment strategies might be challenged. Looking at indications such as Crohn's Disease, a capsule with a panintestinal view can help to assess mucosal healing and thus treat to target. There are lots of different methodologies available and colorectal endoscopy will continue to be the final solution to combine diagnostics with interventions including taking a biopsy. Nevertheless, a pan-intestinal tool as an SBC capsule might strengthen cost effectiveness by replacing multiple tests such as diagnostic endoscopy accompanied by MRI plus Ultrasound. Much more this helps to enhance health-related quality of life for those who suffer.

In the review "Update on Electronic Health Records", Kinberg reveals the opportunities of "e-health" for clinical research in gastroenterology, especially when considering diseases like colorectal cancer and IBD which are of special interest due to its costs, complexity and progressive course (50). The implementation of an "e-capsule service" as described above should open new possibilities for research and treatment. Especially the latter can be addressed when combining it with the pan-intestinal "Crohn's capsule" that might display the treatment effectiveness of Crohn's lesions over time (51).

In Scotland, NHS Highland is piloting a new scheme aimed at combining specialist nursing skills with "smartphone technology" to help improve the quality of life of IBD patients (52). The aim is to track their health status on a regular basis and potentially predict when they might become unwell. Related to this kind of e-health innovation, a diagnostic approach that can be performed outside a clinic definitely looks promising. Teleradiology (53) shows how it can work.

\section{Conclusions}

Video capsule is a highly innovative product which is globally available for a majority of patients, supplementing the toolbox for GI disease detection. The technology is embedded into clinical or ambulant work flow of Gastroenterologists. However, capacities are limited and patients are queuing up. The question is whether individuals with a low risk of severe findings or those who need to assess the course of their disease should be allowed to swallow the capsule where ever it suits them. By all measures, offering a remote capsule service with centralized reading is the next logical step to provide the technology in the short-term to doctors who need a diagnostic tool. That would help to refer all those patients to GIs who need a follow-up. GIs can then focus on interventions, treatment and clinical research of diseases.

Aside from new developments and features, a key aspect to future success of the current video capsule is automation. Instead of individual reading-even if centralizedartificial intelligence could take over describing the level of cleansing, as well as the kind of findings and its precise location. In a future unit, this information would be send to the endoscopic screen to guide next steps.

\section{Acknowledgements}

I would like to express my gratitude to Dirk Stutz, e-learning Manager, ThyssenKrupp Hamburg, and Jon Hill, Independent IT Consultant \& currently a postgraduate at the Usher Institute in Edinburgh, for the valuable inspirational discussions and proofreading.

\section{Footnote}

Conflicts of Interest: T Nowak consults CorporateHealth International ApS, as Director Medical Affairs.

\section{References}

1. Denzer UW, Rösch T, Hoytat B, et al. Magnetically guided capsule versus conventional gastroscopy for upper 
abdominal complaints: a prospective blinded study J Clin Gastroenterol 2015;49:101-7.

2. Gu H, Zheng H, Cui X, et al. Maneuverability and safety of a magnetic-controlled capsule endoscopy system to examine the human colon under real-time monitoring by colonoscopy: a pilot study Gastrointest Endosc 2017;85:438-43.

3. Hale MF, Drew K, Sidhu R, et al. Does magnetically assisted capsule endoscopy improve small bowel capsule endoscopy completion rate? A randomised controlled trial Endosc Int Open 2016;4:E215-21.

4. ASGE Technology Committee, Wang A, Banerjee S, et al. Wireless capsule endoscopy. Gastrointest Endosc 2013;78:805-15.

5. Yung DE, Sykes C, Koulaouzidis A. The validity of suspected blood indicator software in capsule endoscopy: a systematic review and meta-analysis. Expert Rev Gastroenterol Hepatol 2017;11:43-51.

6. Monteiro S, Boal Carvalho P, Dias de Castro F, et al. Capsule Endoscopy: Diagnostic Accuracy of Lewis Score in Patients with Suspected Crohn's Disease. Inflamm Bowel Dis 2015;21:2241-6.

7. Hosoe N, Watanabe K, Miyazaki T, et al. Evaluation of performance of the Omni mode for detecting video capsule endoscopy images: A multicenter randomized controlled trial Endosc Int Open 2016;4:E878-E882.

8. Farhadi H, Nadimi ES, Atai J, et al. A novel adaptive localization technique for wireless capsule endoscopy. Surgical Endoscopy 2015;29:S411.

9. Spada C, Pasha SF, Gross SA, et al. Accuracy of First- and Second-Generation Colon Capsules in Endoscopic Detection of Colorectal Polyps: A Systematic Review and Meta-analysis. Clin Gastroenterol Hepatol 2016;14:1533-1543.e8.

10. Leighton JA, Rex DK. A grading scale to evaluate colon cleansing for the PillCam COLON capsule: a reliability study. Endoscopy 2011;43:123-7.

11. Gluck N, Shpak B, Brun R, et al. A novel prepless $\mathrm{X}$-ray imaging capsule for colon cancer screening. Gut 2016;65:371-3.

12. Malagelada C, Drozdzal M, Seguí S, et al. Classification of functional bowel disorders by objective physiological criteria based on endoluminal image analysis. Am J Physiol Gastrointest Liver Physiol 2015;309:G413-9.

13. Saad RJ. The Wireless Motility Capsule: a One-Stop Shop for the Evaluation of GI Motility Disorders. Curr Gastroenterol Rep 2016;18:14.

14. Pennazio M, Spada C, Eliakim R, et al. Small-bowel capsule endoscopy and device-assisted enteroscopy for diagnosis and treatment of small-bowel disorders: European Society of Gastrointestinal Endoscopy (ESGE) Clinical Guideline. Endoscopy 2015;47:352-76.

15. Rockey DC. Occult and obscure gastrointestinal bleeding: causes and clinical management. Nat Rev Gastroenterol Hepatol 2010;7:265-79.

16. Albert JG, Fechner M, Fiedler E, et al. Algorithm for detection of small-bowel metastasis in malignant melanoma of the skin. Endoscopy 2011;43:490-8.

17. Marelli L, Jaboli FM, Jackson L, et al. A pilot study comparing ESO-2 capsule endoscopy with conventional upper endoscopy for the assessment of uncomplicated heartburn and dyspepsia. Frontline Gastroenterology 2013;4:96-101.

18. Spada C, Hassan C, Sturniolo GC, et al. Literature review and recommendations for clinical application of Colon Capsule Endoscopy. Dig Liver Dis 2011;43:251-8.

19. Carter D, Eliakim R. PillCam colon capsule endoscopy (PCCE) in colonic diseases. Ann Transl Med 2016;4:307.

20. Nogales Ó, García-Lledó J, Luján M, et al. Therapeutic impact of colon capsule endoscopy with PillCam ${ }^{\mathrm{TM}}$ COLON 2 after incomplete standard colonoscopy: a Spanish multicenter study. Rev Esp Enferm Dig 2017;109:322-7.

21. Saurin JC, Ponchon T, Gay G, et al. French multicentric experience of colon capsule endoscopy in real practice: Primary results of the colon capsule endoscopy observatory "ONECC". United European Gastroenterol J 2013;1:A492.

22. Spada C, Hassan C, Galmiche JP, et al. Colon capsule endoscopy: European Society of Gastrointestinal Endoscopy (ESGE) Guideline. Endoscopy 2012;44:527-36.

23. FDA expands indication for PillCam Colon 2 capsule (Apr 2016). Available online: https://www.healio. com/gastroenterology/interventional-endoscopy/ news/online/\%7Bbe015b5b-0375-413a-9c4fabf60bc3ac71\%7D/fda-expands-indication-for-pillcamcolon-2-capsule

24. Japan's Pharmaceuticals \& Medical Devices Agency approved the capsule for diagnosing colonic disease instead of a colonoscopy (Jul 2013). Available online: https://www. globes.co.il/en/article-1000862229

25. Boal Carvalho P, Rosa B, Dias de Castro F, et al. PillCam COLON 2 in Crohn's disease: A new concept of panenteric mucosal healing assessment. World J Gastroenterol 2015;21:7233-41.

26. Oliva S, Di Nardo G, Hassan C, et al. Second-generation colon capsule endoscopy vs colonoscopy in pediatric ulcerative colitis: a pilot study. Endoscopy 2014;46:485-92 . 
27. Flamant M, Trang C, Maillard O, et al. The prevalence and outcome of jejunal lesions visualized by small bowel capsule endoscopy in Crohn's disease. Inflamm Bowel Dis 2013;19:1390-6.

28. Leighton JA, Helper DJ, Gralnek IM, et al. Comparing diagnostic yield of a novel pan-enteric video capsule endoscope with ileocolonoscopy in patients with active Crohn's disease: a feasibility study. Gastrointest Endosc 2017;85:196-205.e1.

29. Hall B, Holleran G, Costigan D, et al. Capsule endoscopy: High negative predictive value in the long term despite a low diagnostic yield in patients with suspected Crohn's disease. United European Gastroenterol J 2013;1:461-6.

30. Bandorski D, Kurniawan N, Baltes P, et al. Contraindications for video capsule endoscopy. World J Gastroenterol 2016;22:9898-908.

31. Rosa B. Capsule Endoscopy in Portugal. Ann Transl Med 2017;5:200.

32. Korman LY, Delvaux M, Gay G, et al. Capsule endoscopy structured terminology (CEST): proposal of a standardized and structured terminology for reporting capsule endoscopy procedures. Endoscopy 2005;37:951-9.

33. Faigel DO, Baron TH, Adler DG, et al. ASGE Guideline: guidelines for credentialing and granting privileges for capsule endoscopy. Gastrointest Endosc 2005;61:503-5.

34. Sidhu R, McAlindon ME, Davison C, et al. Training in Capsule Endoscopy: Are We Lagging behind? Gastroenterol Res Pract 2012;2012:175248.

35. Capsule Endoscopy Training Program. Available online: http://www.capsulecourse.com/

36. The Royal Free PillCam Academy. Available online: https://www.pillcamacademy.com/

37. Video capsule endoscopy hands-on courses. Available online: http://www.kapselklub.de/

38. WEO: Video Capsule Endoscopy Training (VCE). Available online: http://www.worldendo.org/education/ video-capsule-endoscopy-training-vce/

39. Fernandez-Urien I, Panter S, Carretero C, et al. International core curriculum for capsule endoscopy training courses. Endosc Int Open 2017;5:E526-E538.

40. Watabe H, Nakamura T, Yamada A, et al. Assessment of an electronic learning system for colon capsule endoscopy: a pilot study. J Gastroenterol 2016;51:579.

41. Rabinovitz R. Online E-Learning Course: An innovative, new training tool for reading colon capsule endoscopy videos. Available online: http://www.legrandmetier.fr/wpcontent/uploads/2015/04/Online-e-learning-course.pdf

42. Bowel cancer Australia: Doctors concerned growing waiting lists for colonoscopies could prove fatal (Jan 2017). Available online: https://www.bowelcanceraustralia.org/latest-news/ bowel-cancer-doctors-concerned-growing-waiting-lists-forcolonoscopies-could-prove-fatal-abc-news

43. AOK Bavaria and partners: a German model project to use colon capsule endoscopy for CRC screening. Available online: http://darmkapsel.de/aok/die-partner/

44. Khoury M, Bredow L, Khoury F, et al. Enabling tandem procedures of colonoscopy following Colon Capsule Endoscopy in Colorectal Cancer Screening. P0859 UEGW 2015.

45. Gaunsbæk H. Camera pill (colonic capsule endoscopy) for screening of colorectal cancer. Available online: http:// cimt.dk/en/projects/innovation/pillcam/\#.WfUAVIVOJBo

46. Colonic Capsule Endoscopy (CCE) for Screening of Neoplasm's A Pilot Study. ClinicalTrials.gov Identifier: NCT02303756

47. Ofstead CL, Wetzler HP, Heymann OL, et al. Longitudinal assessment of reprocessing effectiveness for colonoscopes and gastroscopes: Results of visual inspections, biochemical markers, and microbial cultures. American Journal of Infection Control 2017;45:e26-e33.

48. Guarini A, De Marinis F, Hassan C, et al. Accuracy of trained nurses in finding small bowel lesions at video capsule endoscopy. Gastroenterol Nurs 2015;38:107-10.

49. Koprowski R. Overview of technical solutions and assessment of clinical usefulness of capsule endoscopy. Biomed Eng Online 2015;14:111.

50. Kinberg S. Update on Electronic Health Records: Opening a new era for clinical research in gastroenterology (Jan 2017). Available online: http://www.gastroendonews.com/ Review-Articles/Article/01-17/Update-on-ElectronicHealth-Records-/39044

51. Hausmann J, Schmelz R, Walldorf J, et al. Pan-intestinal capsule endoscopy in patients with postoperative Crohn's disease: a pilot study. Scand J Gastroenterol 2017;52:840-5.

52. NHS Highland pilots Smartphone technology for Crohn's disease (Nov 2013). Available online: http://www. nhshighland.scot.nhs.uk/News/Pages/NHSHighlandpilots SmartphonetechnologyforCrohn'sdisease.aspx

53. ESR European Society of Radiology white paper on teleradiology: an update from the teleradiology subgroup. Insights Imaging 2014;5:1-8.

Cite this article as: Nowak T. A global perspective on capsule endoscopy. Ann Transl Med 2017;5(21):422. doi: 10.21037/ atm.2017.10.20 